\title{
Principios Prácticos y Funcionales en Situaciones de Muerte y Duelo para Profesionales de la Educación
}

\author{
Practical and Functional Principles in Situations of Death and \\ Mourning for Professionals of Education
}

\author{
Anabel Ramos-Pla ${ }^{1 *}$ \\ Joaquín Gairín ${ }^{1}$ \\ Ramón Camats ${ }^{2}$ \\ ${ }^{1}$ Universitat Autònoma de Barcelona, España \\ ${ }^{2}$ Universitat de Lleida, España
}

\begin{abstract}
Actualmente, la sociedad está viviendo una regresión sobre la concepción de la muerte. La idea de morir se asocia a miedo, tristeza y angustia. La presente aportación proporciona a los profesionales de la educación una serie de pautas para acompañar a los niños y adolescentes en los procesos de muerte y duelo. Se apuesta por la inclusión de la muerte en el currículum escolar como eje para la enseñanza de valores y la normalización de la muerte como un hecho que forma parte de la vida. El método utilizado para llevar a cabo la extensa búsqueda bibliográfica ha sido, principalmente, la búsqueda y el seguimiento de la temática que nos ocupa en diferentes bases de datos nacionales e internacionales. De este modo, el método utilizado para analizar las referencias bibliográficas encontradas ha sido, principalmente: la idoneidad a la temática, la calidad de la revista, el año de publicación y la importancia de la autoría. El propósito último de este estudio es concebir y tratar la muerte y el duelo desde la perspectiva sistemática que la intervención pedagógica aporta, lejos de los procesos de improvisación. Es importante remarcar que este documento no pretende ser una generalización, si no que cada profesional de la educación que quiera llevar a cabo una pedagogía de la muerte (ya sea preventiva, paliativa o ambas), debe tener en cuenta el contexto. Asimismo, la prospectiva de futuro de la pedagogía de la muerte es muy positiva, ya que es un tema emergente y necesario en el terreno pedagógico.
\end{abstract}

Descriptores: Educación; Muerte; Formación; Docente; Innovación educacional.

Currently, society is experiencing a regression on the conception of death. The idea of dying is associated with fear, sadness and anguish. This contribution provides education professionals with a series of guidelines to accompany children and adolescents in the processes of death and grief. It is committed to the inclusion of death in the school curriculum as an axis for the teaching of values and the normalization of death as a fact that is part of life. The method used to carry out the extensive bibliographic search has been, mainly, the search and follow-up of the subject matter that occupies us in different national and international databases. In this way, the method used to analyse the bibliographical references found was mainly: the suitability of the subject, the quality of the journal, the year of publication and the importance of authorship. The ultimate purpose of this study is to conceive and treat death and grief from the systematic perspective that pedagogical intervention provides, far from the processes of improvisation. It is important to note that this document does not intend to be a generalization, but rather that each education professional who wants to carry out a death pedagogy (be it preventive, palliative or both), must take the context into account. Likewise, the prospective of the future of the pedagogy of death is very positive, since it is an emerging and necessary subject in the pedagogical field.

Keywords: Education; Death; Training; Teachers; Educational innovations.

*Contacto: anabelrms@gmail.com

Recibido: 3 de agosto 2017

$1^{\text {a }}$ Evaluación: 15 de octubre 2017

ISSN: $1696-4713$

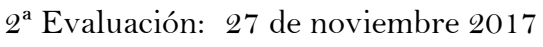

www.rinace.net/reice/

Aceptado: 10 de diciembre 2017

revistas.uam.es/reice 


\section{Contextualización del tabú de la muerte: Una regresión real}

La cultura occidental parte de unos valores donde la persona como individuo tiene mucha importancia y donde se intenta preservar la vida por encima de todo (Fernández, 2013), incluso sin tener en cuenta la calidad. Se han producido multitud de adelantos tecnológicos y se ha aumentado la longevidad de la población, pero, a su vez, se han ido perdiendo algunos valores comunitarios y espirituales. Esquerda y Agustí (2010) añaden que vivimos en una sociedad de culto al cuerpo, la inmediatez y la tecnología omnipotente. Todo ello ha generado una falsa creencia de control que deja de ser real cuando se cae en la cuenta que la muerte no es controlable ni evitable. Además, la consecuencia directa de estos hechos es que los humanos han pasado de concebir la muerte como algo normal en la vida, ya que tarde o temprano sucede, a transformarlo en un hecho privado y oculto. Así, se espera morir de forma inconsciente y rápida, sin poder despedirse.

La muerte provoca sufrimiento y, por tanto, origina emociones que no deseamos. Por este motivo, se evita hablar de la muerte y se elude de la vida cotidiana convirtiéndola en un tabú. Este hecho no favorece la elaboración de los conceptos de muerte y duelo en contextos vitales diversos y con total normalidad. Desde este punto de vista, morir se convierte en algo terrorífico porque supone la pérdida de todo y, además, es una expresión de nuestra debilidad. Por ello, en general no se habla de la muerte y vivimos sin pensar que la muerte forma parte de la vida (Díaz, 2004).

En nuestra sociedad de desarrollo y de consumo existen dos grandes posiciones o discursos sobre la muerte (Herrán y Cortina, 2006): una mayoritaria de rechazo segregador o de refugio en la superficialidad y otra, más culta, de interés integrador y educativo. Contrariamente a la concepción occidental sobre la muerte, podemos observar la situación de las comunidades orientales. Fernández (2013) apunta que se vive la muerte como una fase más de la propia existencia; es decir, morir supone un traspaso y no un final y, además, la muerte está muy presente en su cultura cotidiana. Podríamos preguntarnos, al respecto, por qué no se adoptan rasgos de las culturas orientales para vivir la muerte de la misma forma que se vive la vida.

Cuando muere alguien cercano, apartamos a los niños y adolescentes de esta situación para protegerlos del dolor (Herranz, 2016) o al menos es la idea con la que se lleva a cabo este acto de separación. Asimismo, realizando actos (o, mejor dicho, no-actos) como el que acabamos de mencionar, olvidamos que los niños y adolescentes también sienten la pérdida del ser querido a su manera. Por lo tanto, también están elaborando su duelo. Si los adultos omiten el sufrimiento y el dolor hacia los más pequeños, no se les ayuda a expresar sus sentimientos y emociones. Debemos tener en cuenta que el sufrimiento forma parte de la vida y, por tanto, se debe pasar de forma resiliente; además, si no se explica la verdad y no se responden las dudas de los más pequeños, se pueden crear fantasías sobre la muerte y todo lo que puede repercutir en las vivencias personales se magnifica innecesariamente. Hechos de este tipo, potencian todavía más el tabú que precede la muerte en la sociedad occidental.

Herrán y Cortina $(2006,2008)$ añaden que la muerte como cuestión educativa es un factor de máxima resistencia porque no tiene tradición profesional en la educación y, además, las creencias existentes en otros ámbitos incitan a la negatividad y el miedo. Los mismos autores abogan que estamos viviendo un fenómeno de cultura anestesiada y, por tanto, de 
anestesia de la muerte. Este hecho solo omite y tapona la muerte, evitando y dificultando que los niños y adolescentes elaboren su propio duelo. ¿Con qué derecho omitimos un proceso natural, como es la muerte, a los niños y adolescentes? ¿Por qué dificultamos la elaboración positiva de su propio duelo?

Kübler-Ross (2003) en sus estudios y experiencia con pacientes moribundos afirmó que, si se vive la muerte como un proceso de transformación y cambio y se usa este momento para enriquecerse uno mismo de forma espiritual y maduración, el resultado será un duelo muy positivo. En definitiva, abordar la muerte, tanto desde la propia sociedad como en el ámbito educativo, es todo un reto, un tabú moderno que es necesario romper para normalizar el contenido afectivo que tiene la muerte para cualquier persona (Esquerda y Agustí, 2010).

\section{Procedimiento}

El método utilizado para obtener las referencias bibliográficas citadas a lo largo del presente artículo son fruto de la investigación y seguimiento de diferentes bases de datos internacionales (ERIC) y nacionales (Dialnet y repositorios de diversas universidades españolas). De este modo, el método para llevar a cabo el análisis de la bibliografía fue un seguimiento sistemático de todas aquellas publicaciones publicadas en los últimos 5 años, enfatizando toda aquella investigación que fuera del presente año. Además, se siguieron otros criterios como la idoneidad con la temática que nos ocupa (enfocando sobre todo a aquellas publicaciones que hicieran referencia a la pedagogía de la muerte de forma práctica), la calidad científica de las publicaciones y la importancia de la autoría (enfatizando aquellos autores en los cuales se fundamentan las bases pedagógicas actuales de la pedagogía de la muerte).

\section{Principios básicos para comunicar la muerte de un ser querido a un niño}

Cuando se habla de la comunicación de la defunción de un ser querido a un niño o adolescente y sobre qué es lo más recomendable a realizar ante tal situación, se deben tener en cuenta diferentes factores. No es una situación fácil y, por tanto, es necesario que los profesionales de la educación reciban información para no actuar de forma improvisada. Proponemos posteriormente algunas líneas de actuación para afrontar el proceso de comunicación de la muerte de un ser querido y todo lo que esa comunicación supone. Hay que considerar que estas líneas de actuación no son homogéneas y aplicables a todos los casos de comunicación de una muerte; no obstante, sí que se trata de intervenciones que son consideradas válidas desde el punto de vista de la funcionalidad que tienen.

\subsection{La importancia de la formación del profesorado en relación a la pedagogía de la muerte}

Para poder llevar a cabo la pedagogía de la muerte, se necesita una formación pedagógica profunda y concreta con relación a la conciencia de la realidad sobre la finitud humana (Benlloch et al., 2015; Cantero, 2013; Colomo, 2016; Colomo y De Oña, 2014; Ransanz, 2015; Rodríguez Herrero, Herrán y Cortina, 2015; Serra, 2014; Shumba, Moyo y Rembe, 2015; entre otros), necesaria porque la gran mayoría de profesionales de la educación tiene un gran desconocimiento sobre la temática y, curiosamente, debe intervenir con alumnos. 
El hecho de tener un conocimiento y aprendizaje correcto y adecuado sobre la muerte y la buena elaboración del duelo es consecuencia directa del desarrollo de un mayor sentido de la humanidad, que nos ayuda a mejorar como seres humanos (González y Herrán, 2010). Así, la pedagogía de la muerte tiene un gran valor formativo, ya que su finalidad última es el desarrollo integral de los discentes (Cortina y Herrán, 2005; Ransanz, 2015).

Estudios realizados por Colomo (2016) mediante la implementación de una propuesta didáctica en estudios universitarios ofrecieron, en este sentido, resultados destacados. La propuesta se realizó con alumnos del Máster Universitario en Formación del Profesorado en Educación Secundaria Obligatoria, Bachillerato, Formación Profesional y Enseñanza de Idiomas dentro de la asignatura Educación emocional y habilidades sociales, en la Universidad Internacional de Valencia (curso 2014/15). Esta asignatura pretendía conocer y profundizar en el conocimiento del duelo utilizando los cuentos como herramienta pedagógica terapéutica. La primera conclusión que remarcó la importancia de una formación en pedagogía de la muerte, por ser una temática omnipresente, ineludible y de indispensable abordaje en el ámbito educativo; también, porque no se puede enseñar o acompañar en contenidos que se desconocen por no haber tenido una experiencia directa o por no haber recibido formación específica en la temática. La segunda conclusión fue que el cuento es un recurso pedagógico óptimo para abordar la educación sobre la muerte porque permite trabajar el control y la gestión emocional de forma preventiva y paliativa de la muerte y el duelo.

También podemos encontrar otras experiencias de pedagogía de la muerte dentro del ámbito universitario, como las de Song y Kim (2015) en Corea del Sur, Zhang y Li (2016) en China o la de Corr (2016), quien realizó cursos de formación en diversas instituciones de Canadá y en universidades de los Estados Unidos (Southern Illinois University Edwardsville, University of Maryland, Bowling Green State University, University of Akron y University of Central Oklahoma). A estos cursos asistía una gran diversidad de estudiantes: profesionales que se encontraban habitualmente con la muerte (médicos, enfermeras, maestros, etc.), personas a quien hacía relativamente poco que se les había muerto un ser querido o bien querían prepararse para una futura pérdida y, finalmente, personas que asistía por simple curiosidad. Normalmente, Corr (2016) estructuraba los cursos en diez contenidos: educación sobre la muerte, el morir y el duelo; cambiando los encuentros con la muerte; cambiando las actitudes respecto la muerte; el sistema estadounidense de muerte; prácticas relacionadas con la muerte; patrones culturales de la muerte; el duelo; perspectivas de desarrollo; conceptos legales y cuestiones morales. La evaluación realizada por el mismo autor fue del todo positiva, ya que afirmó que los estudiantes aprenden a entender y a ejercer el control en sus vidas; aprenden de las limitaciones humanas, de su propia individualidad como seres sociales; comprenden en profundidad la vulnerabilidad y la resiliencia humanas e interiorizan la importancia de la búsqueda del sentido de la vida.

No solo debemos actuar como podamos en las situaciones de muerte y duelo en los centros educativos, sino que debemos hacerlo como sabemos, de forma programada y justificada. Ramos Álvarez (2015) alega que las posibilidades de fracaso en las intervenciones educativas con estudiantes en duelo son muy altas por la falta de cultura pedagógica al respecto.

Cortina y Herrán (2011) proponen realizar una serie de acciones y actividades formativas con los objetivos de favorecer que el profesorado adquiera un mayor conocimiento sobre 
la muerte y que repercuta directamente en el cambio conceptual y en la pérdida del tabú, para poder llevar a cabo esta formación. Es importante concienciar a los profesionales de la educación en un sentido existencial y, a su vez, de la importancia de la pedagogía de la muerte, tanto en el sentido preventivo como paliativo teniendo en cuenta las emociones (Cantero, 2013). Es decir, debemos examinarnos a nosotros mismos hasta llegar a encarar el hecho de morir, ya que comprender la muerte supone una inyección de sentido existencial de la propia vida.

No se puede tratar un tema tan complejo como la muerte si antes no se ha reflexionado e interiorizado como tema transversal de la práctica educativa normalizada. Para poder tratar el enfoque preventivo de la muerte, hará falta que la formación de los profesionales de la educación cumpla las siguientes pautas (Herrán y Cortina, 2009):

- Formación de los equipos directivos: cursos de formación continua, grupos de discusión y mesas redondas, grupos de investigación-acción y asesoramiento por parte de expertos en la temática.

- Formación de maestros y familias: charlas, mesas redondas, paneles de expertos, seminarios, entrevistas públicas, role-playing, etc.

Debemos recordar que, como profesionales de la educación, debemos afrontar nuestros prejuicios, miedos, etc., dado que proyectamos todas estas emociones y sentimientos en los niños y jóvenes. Cambiar estas actitudes resultará una tarea compleja porque no suele producirse de inmediato, sino que se requiere un periodo de latencia (Aradilla, 2004; Baum, 2003; Rodríguez Fernández, 2000). Para poder llevar a cabo este proceso tan complejo de deconstrucción de esquemas e ideas preconcebidas, se debe hacer un trabajo previo de elaboración de los propios duelos y de replanteamiento de las experiencias pasadas en referencia a la muerte. Si no se han elaborado correctamente los duelos o siempre se ha escondido la muerte, difícilmente se podrá enseñar y acompañar a los infantes tanto en una pedagogía preventiva sobre la muerte como en la paliativa. Cabe recordar que los dos tipos de enseñanzas se ven organizadas y englobadas dentro de la pedagogía de la muerte o de la educación para la muerte.

Por todo lo mencionado, es necesario potenciar los espacios de reflexión con los docentes y otras tipologías de profesionales (psicopedagogos, pedagogos, psicólogos, enfermeros, familiares, etc.) (Dussel y Southwell, 2007). De este modo se puede reflexionar desde diferentes miradas y perspectivas, desde la teoría y la práctica para, así, aprender por y para los demás.

\subsection{Recomendaciones para comunicar una defunción}

En primer lugar, es recomendable que la noticia de la muerte de un ser querido la dé una persona emocionalmente próxima al niño o adolescente (Poch y Herrero, 2003), de tal manera que el entorno sea lo más seguro posible. A veces, esta propuesta no siempre es posible, ya que, por ejemplo, ante la muerte de la pareja o un hijo, la madre o padre superviviente ha de añadir a su sufrimiento y dolor la responsabilidad de tener en cuenta al otro hijo. Únicamente cuando sucedan hechos de tal calibre, donde los padres no son capaces de dar la noticia al niño o adolescente, debe entrar en acción la figura del profesional de la educación: el maestro tutor, el maestro o el psicopedagogo.

En el caso de la muerte por suicidio, será necesario extremar el cuidado en el momento de comunicarlo al niño o adolescente. Debemos buscar una causa explicativa y adecuada a su 
edad, debiendo comunicarle que ha sido un accidente, que se ha caído del balcón, etc. A los más pequeños, podemos decirles que ha estado muy enfermo y no ha podido vivir más. Cabe añadir que a los adolescentes podemos ofrecerles la noción de voluntariedad, pero esta concepción se vinculará al intenso sufrimiento mental y a la falta de recursos personales.

Es necesario comunicar la defunción de un ser querido con palabras sencillas y sinceras, de forma dulce y con afecto (Kroen, 1996). En este sentido, esta autora proporciona una serie de recomendaciones para docentes y psicopedagogos, familias, niños y adolescentes que ampliamos a continuación.

- En primer lugar, no tener miedo a utilizar las palabras "muerte" o "ha muerto". El profesional de la educación debe tener una concepción de la muerte como parte de la vida, considerando que, si tiene miedo o le producen rechazo las situaciones de muerte y duelo, transmitirá estas expectativas al niño o adolescente. Por ello, adquiere gran importancia la conciencia profunda por parte del profesorado y los psicopedagogos de las situaciones de muerte y duelo y todo lo que ello conlleva.

- Es necesario desmitificar el estereotipo de que los niños son demasiado pequeños para comprender una muerte. Sienten y lloran la muerte de un ser querido y experimentan emociones muertes; evidentemente no lo harán de forma adulta, pero su edad no exime el hecho de que sientan la pérdida. Los adolescentes sienten y experimentan la muerte y el duelo de una forma más parecida a la de un adulto por lo que será necesario que los profesionales de la educación adapten los contenidos sobre la muerte y el duelo en función de la etapa evolutiva y las necesidades de los niños y adolescentes; es decir, no se omitirá el hecho de morir, pero sí que se adaptará.

- Usar con los más pequeños adverbios del tipo "papá ha estado muy, muy, muy enfermo y ha muerto". Emplear múltiples "muy" implica que la mayoría de seres vivos disfrutamos de una vida longeva, evitando de esta forma, al niño el miedo a morir en el mismo momento. En función de la edad y la etapa evolutiva del niño o adolescente, se irán eliminando progresivamente estos adverbios y se irán dando más dosis de realidad. En el caso que muera el ser querido de un adolescente, se le puede comunicar el motivo de la defunción, por ejemplo, que ha padecido cáncer o ha tenido un accidente de tráfico.

- Explicar de forma sencilla y con pocas palabras que el ser querido ha muerto. Fernández (2013) añade que debemos entender y respetar el ritmo y estilo comunicativo de cada individuo, teniendo siempre en cuenta la escucha activa, la veracidad y la adecuación. En definitiva, los profesionales de la educación no deben dar muchos rodeos en el momento de comunicar una muerte ni inventarse "nuevos lugares" para decir donde se encuentra el ser querido, ya que ni está en el cielo, ni se encuentra en una estrella, sino que ha muerto. En este sentido, es importante explicarles a los más pequeños la muerte biológica, es decir, el paro de las funciones vitales. Debemos recordar los estadios evolutivos y el hecho que hasta los siete u ocho años (etapa de las operaciones concretas) no empiezan a comprender el lenguaje simbólico.

- Evitar eufemismos como "pérdida", "se lo han llevado", "ha desaparecido", "se ha quedado dormido" o "se ha ido de viaje". Estos eufemismos alimentan el miedo 
de los niños a ser abandonados y a quedarse dormidos. Debemos prevenir la creación y surgimiento de la ansiedad y la confusión.

- Si el enfermo es el niño o adolescente, debemos hacerle partícipe lo más pronto posible de este hecho, en el nivel que él pueda comprenderlo. De hecho, seguramente, se lo intuía desde el inicio de su enfermedad y reivindicamos la innecesariedad de omitir la realidad a los niños y adolescentes, y el pensar que todavía no pueden entender la muerte.

\subsection{La importancia del acompañamiento educativo en procesos de muerte y duelo}

Diversos autores han hablado de la importancia del acompañamiento empático hacia los niños y adolescentes. Herrán y Cortina (2008) apelan que acompañar al otro desde la perspectiva empática equivale a dejarse llevar, de forma automática, de tal forma que el proceso se coloque en función de las necesidades de la persona, sus circunstancias y del momento que se indica, incluso según nuestra personalidad. En esta línea, Bowlby (1998) añade que poder ser acompañado en todo momento por alguien cercano que comprenda y ayuda a poner palabras al dolor que se siente después de la pérdida de un ser querido es un hecho clave para asegurar un proceso de duelo positivo.

Debemos considerar que la importancia del proceso del acompañamiento empático no depende de saber cómo explicar, sino de cómo debemos acompañar al niño o adolecente. Asimismo, debemos comunicar al alumno nuestra intención educativa y orientadora para saber que comprendemos su dolor y que sepa que lo ayudaremos. De esta forma, sabrá que no estará solo delante del reto más difícil que nos impone la vida: la muerte.

Cabe añadir que no se puede poner remedio a la pérdida ni a los sentimientos que causa, pero sí podemos ayudar al niño o adolecente a expresar las emociones que está experimentado, ya que pueden ser nuevas para él. Además, podemos responder sus dudar, clarificar malentendidos y calmar miedos irracionales. Si se comparten los miedos, estos tienden a disminuir.

Turner (2004) añade que es de vital importancia ayudar al niño o adolescente a tomar conciencia de que la muerte de su ser querido no ha sido su culpa. Los menores pueden concebir la muerte de un ser querido como consecuencia de un acto cometido por ellos; es decir, un niño puede pensar que su madre ha muerto porque la semana anterior hizo alguna travesura. Por este motivo, debemos darle pie a que exprese todo aquello que sienta (tristeza, rabia, miedo, confusión, preocupación, entre otras emociones) para, así, poder acompañar su proceso de duelo. De este modo, podemos ayudar a retirar progresivamente al niño o adolescente, la idea de culpabilidad que pueda tener sobre la muerte de su ser querido.

\section{Orientaciones prácticas y funcionales para acompañar en los procesos de muerte y duelo}

Comentamos, a continuación, diferentes técnicas y estrategias para acompañar desde el ámbito educativo a los niños y adolescentes en los procesos de muerte y duelo, compartiendo con Herrán y Cortina (2008) la idea de la importancia de la actuación desde la flexibilidad. Existen tres premisas a tener en cuenta en el momento de acompañar a los niños y adolescentes: la primera es que el alumno podrá contar con el apoyo del profesional de la educación, ya sea maestro tutor, profesor o psicopedagogo, para avanzar en su 
proceso de duelo; la segunda es que ninguna de las respuestas del alumno será censurada, adjetivada o despreciada; además, no debemos mentir, interrogar, juzgar, imponer, evadir ni realizar interpretaciones sobre sus comentarios; por último, las anteriores premisas generarán una buena base de enriquecimiento para el intercambio humano.

\subsection{Técnicas para la enseñanza y tratamiento de la muerte y el duelo}

Algunas de las actuaciones que se pueden llevar a cabo con los alumnos que se encuentren en situaciones de muerte y duelo (Herrán y Cortina, 2006, 2008; Poch y Herrero, 2003) o desde una perspectiva preventiva, anticipando el concepto de muerte antes que suceda, son las siguientes:

- Narrativas: cartas de despedida a la persona o animal querido que ha muerto o a una emoción o pensamiento que el niño o adolescente considere relevante en aquel instante. Además, puede confeccionar un diario con sus vivencias sobre la muerte y el duelo.

- Trabajos con poemas y metáforas de la muerte y el duelo.

- Existen actividades que se pueden realizar antes y después del acontecimiento trágico. Algunas de ellas son los rincones de juego (rincón de los animales extinguidos, rincón de los miedos, etc.), centros de interés (la nieve y los riesgos que comporta, el otoño con la muerte de las hojas y la vida latente), comentarios de las consecuencias de los actos terroristas, significatividad de las catástrofes naturales, etc.

- Pequeñas investigaciones sobre cuánto vive cada ser vivo. Por ejemplo, la longevidad de un perro no es la misma que la de una persona.

- Proyectos de vida en la naturaleza: actividades de reciclaje, reconstrucción ambiental, primeros auxilios, etc.

- Talleres cooperativos donde la muerte no se niegue. Por ejemplo, se puede analizar un periódico donde aparecen muertes en guerras, accidentes de tráfico, etc.

- Participar en fiestas tradicionales como el día de los muertos (uno de noviembre), el entierro de la sardina o el día de los derechos de los niños y adolescentes.

- Talleres de técnicas diversas. Por ejemplo: de cocina, de rebisabuelos donde los alumnos pueden imaginar qué les sucedió o puedes preguntar a sus familiares y plasmarlo en una línea del tiempo.

- Talleres globalizados de teatro, de cuentos, de lecturas dramatizadas, transculturales (diversas formas de entender la muerte desde diferentes puntos de vista teniendo en cuenta la multitud de culturas existentes), de fotografías antiguas, etc.

- Lluvia de ideas sobre el concepto de muerte y duelo donde cada alumno o por grupos deben plantear dudas sobre los dos términos para, posteriormente, realizar un debate.

- Homenajes y días virtuales para las catástrofes hechas por el hombre como pueden ser las guerras, los actos terroristas, etc. 
- Entrevistas públicas colectivas con expertos. Por ejemplo, la asistencia a una charla del médico de un alumno que haya superado una enfermedad grave. También puede ser útil la asistencia a la charla de o un sepulturero.

Desde la perspectiva más innovadora de la pedagogía de la muerte, se apuesta por una propuesta integrada dentro de los proyectos educativos de centro en los cuales la muerte sea una de los elementos vertebradores de las acciones educativas. De este modo, se podría realizar el conjunto de propuestas mencionadas desde una perspectiva integral y transversal de la educación de los niños. Se potenciaría una educación real, desde la perspectiva interpersonal e intrapersonal, donde un hecho real de la vida conduciría al resto de elementos curriculares como la educación para la salud, la filosofía, las ciencias naturales y sociales, la religión, etc.

\subsection{Actividades y juegos para que los niños y adolescentes puedan expresar los sentimientos de rabia respecto la muerte}

En los procesos de duelo, y sobre todo en niños y adolescentes, la rabia es un sentimiento que se experimenta con fuerza y, a menudo, hace sentir mal al que la siente. Los niños y adolescentes pueden canalizar este sentimiento de rabia mediante su cuerpo y, por este motivo, proponemos una serie de actividades y juegos para orientar y encauzar la rabia (Díaz, 2004):

- Juegos y actividades que comporten ensuciar o ensuciarse uno mismo: dibujar con pintura de dedos, modelar barro, etc.

- Tareas que impliquen ejercicio físico y una descarga de energía: correr, practicar deportes, saltar, etc.

- Hacer cosquillas a otra persona.

- Todos los juegos y actividades donde se utilicen materiales que se puedan recortar, pinchar o golpear: perforar dibujos, recortar papeles, utilizar sierras y martillos infantiles, enganchar botones, etc.

- Juegos de lanzamiento de objetos como, por ejemplo, jugar a básquet, voleibol o balonmano.

- Romper o destruir cosas que se han construido previamente, como pisar figuras hechas con plastilina, empujar castillos de cartas, etc. Es de vital importancia vigilar que el alumno no destruya nada antes de haber terminado la figura que haya hecho previamente. Destruir no es terapéutico si esta acción representa una reacción a la frustración.

- Juegos que requieran el sentido competitivo. Asimismo, se debe vigilar que la competencia sea entre un niño o adolescente y un adulto (y no entre dos alumnos de la misma edad) y entre equipos numerosos y equilibrados (mismo número de miembros por equipo y máxima igualdad de fuerzas y habilidades).

Por último, cabe decir que el sentido común es nuestra mejor herramienta. Además, si proponemos actividades demasiado difíciles, solo conseguiremos que el niño o adolescente se frustre, se sienta mal. Por el contrario, si le proponemos tareas demasiado fáciles, se aburrirá. Por este motivo, tal y como hemos ido comentando, es de vital importancia adaptarse a la edad del alumno, a su estadio evolutivo y a su ritmo de trabajo. De esta 
forma, únicamente llevará a cabo actividades que estén adaptadas para él sin que llegue a aburrirse o a frustrarse.

\subsection{Actividades y juegos para promover la autoestima de los niños y adolescentes respecto la muerte de un ser querido}

En el momento de elaborar un duelo, los niños y adolescentes se sienten diferentes a su grupo de iguales. Este sentimiento de diferencia se debe a que el sentimiento de pérdida puede inducirlos a pensar que no son tan importantes como sus compañeros de clase. El maestro, profesor o psicopedagogo puede acompañarlos en este duro proceso de transición y, así, potenciar su autoestima. Algunas de las actividades para conseguir la autoestima pueden ser las siguientes (Díaz, 2004):

- Participar en juegos y actividades educativas que comporten aprender cosas nuevas. Para hacerlo, debemos tener siempre en cuenta la edad y el estadio evolutivo del niño o adolescente.

- Confeccionar objetos u ornamentos para su habitación: carteles con su nombre, ornamentos para las camisetas, etc.

- Realizar actividades creativas que impliquen dibujar, diseñar, construir o inventar historias, cuentos, argumentos, etc.

- Elaborar comidas fáciles, adornar pasteles, preparar bocadillos, etc. De nuevo, debemos tener en cuenta la edad del niño o adolescente y estar alerta a cómo se desenvuelve en la cocina.

Díaz (2004) también recomienda que el niño o adolescente no deje ninguna de sus tareas a medias en el instante que se desanime o encuentre una dificultad. Como profesionales de la educación, debemos ayudarlo y motivarlo para que piense y reflexione sobre las soluciones.

\section{Conclusiones}

Hoy en día se omite la muerte en la vida cotidiana de los niños y adolescentes dificultando la concepción y elaboración de la muerte y el duelo. Por este motivo, desde las instituciones educativas se debe potenciar la enseñanza de la muerte como parte de la vida y el acompañamiento empático como base pedagógica en los procesos de duelo.

La importancia de las figuras del maestro-tutor y del psicopedagogo nos parecen importantes en esta temática y reivindicamos que así se reconozca. Muchas veces, los padres y familiares cercanos de los niños y a los adolescentes no son capaces de comunicarles la realidad por la gran carga emocional que supone la pérdida de alguien próximo. Comunicar la muerte de un ser querido, y más aún si es muy cercano, es una situación muy dura y puede aconsejar que actúe el maestro-tutor o el psicopedagogo, preparado y formado previamente para abordar situaciones como estas. A su vez, debemos recordar que cuando se informa de la muerte de un ser querido a un niño o un adolescente, se debe hacer con palabras simples, afecto y sinceridad. Los niños saben y perciben mucho más de lo que los adultos pueden imaginar.

Cabe añadir que, si se dedican los esfuerzos a la formación inicial del profesorado en relación a la pedagogía de la muerte, se estará llevando a cabo una educación efectiva y significativa. Tal y como hemos ido desarrollando a lo largo del artículo, se debería 
introducir y profundizar sobre la muerte, su tratamiento preventivo y paliativo y el acompañamiento en el duelo durante la formación inicial de los docentes, pedagogos y psicopedagogos. Del mismo modo, es necesario tomar conciencia sobre como los profesionales de la educación debemos actualizarnos continuamente y, con más motivo, en temáticas tan complejas como el abordaje pedagógico de la muerte. Asimismo, se debe alentar a los aprendices de maestro a asistir a seminarios y conferencias sobre la temática, ejercer el voluntariado, realizar cursos, etc.

La aportación presenta diversas estrategias y actividades para que los profesionales de la educación tengan recursos para atender las situaciones de muerte y duelo. Con ello, se pretende potenciar la actuación desde la pedagogía y no desde la improvisación, al mismo tiempo que rechazamos la idea de la no actuación frente a las situaciones y procesos de muerte y duelo por no suponer ningún tipo de finalidad educativa ni terapéutica.

Por último, las actividades de actuación presentadas son propuestas comprobadas y consolidadas por diferentes estudios (Herrán y Cortina, 2006, 2008; Kroen, 1996; Turner, 2004), debiendo considerarlas como ejercicios abiertos, adaptables a cualquier edad, etapa evolutiva y ritmo de aprendizaje. Los maestros y psicopedagogos deben poner en práctica estas actividades para trabajar y tratar la muerte y el duelo desde el conocimiento y la aceptación de la realidad. El humano es un ser finito y, por tanto, este hecho debe ser enseñado y tratado.

Esta recopilación bibliográfica no pretende ser una generalización, pero si una orientación en relación a la pedagogía de la muerte. En este sentido, es importante que la figura del profesional de la educación que pretenda llevar a cabo este tipo de pedagogía, analice las propuestas y las adapte según el contexto y las necesidades del mismo. Por ello, no en todos los centros educativos y con todos los alumnos se pueden llevar a cabo las mismas prácticas educativas.

Actualmente, la pedagogía de la muerte es un tema emergente en el ámbito educativo y una necesidad imperante. Las aportaciones pedagógicas continúan siendo escasas, sobre todo las prácticas reales. Por ello, se podrían realizar investigaciones en contextos reales de la aplicación de métodos citados en el presente estudio.

\section{Referencias}

Aradilla, A. (2004). La muerte una pedagogía emocional. Monitor Educador, 104, 13-16.

Baum, H. (2003). ¿Está la abuelita en el cielo? Cómo tratar la ausencia y la tristeza con los niños. Madrid: Espasa Libros.

Benlloch, M. J., Vazquez, V., Boluda, J. y García Bataller, E. (2015). APS como metodología para trabajar la muerte y el duelo en futuros educadores. En A. Cagnolati y J. L. Hernández Huerta (Coords.), Pedagogía ante la muerte. Reflexiones e interpretaciones en perspectivas histórica y filosófica (pp. 21-27). Salamanca: FahrenHouse.

Bowlby, J. (1998). El aferrament y la pèrdua - 1: L’aferrament. Barcelona: Paidós.

Cantero, M. F. (2013). La educación para la muerte. Un reto formativo para la sociedad actual. Psicogente, 16 (30), 424-438.

Colomo, E. (2016). Pedagogía de la muerte y proceso de duelo. Cuentos como recurso didáctico. REICE. Revista Iberoamericana sobre Calidad, Eficacia y Cambio en Educación, 14(2), 1-15. https://doi.org/10.15366/reice2016.14.2.004 
Colomo, E. y De Oña, J. M. (2014). Pedagogía de la muerte. Las canciones como recurso didáctico. REICE. Revista Iberoamericana sobre Calidad, Eficacia y Cambio en Educación, 12(3), 109-121.

Corr, C. A. (2016). Teaching about life and living in courses on death and dying. OMEGA, 72(2), 174-187.

Cortina, M. (2003). Educar teniendo en cuenta la muerte. Aula de Innovación Educativa, 122, 52-58.

Cortina, M. y Herrán, A. (2005). Cine y educación para la muerte II. Making Of. Cuadernos de Cine y Educación, 36, 20-33.

Cortina, M. y Herrán, A. (2011). Pedagogía de la muerte a través del cine. Madrid: Editorial Humanitas.

Díaz, I. (2004). I ara, on és? Com ajudar els nens i els adolescents a entendre la mort. Barcelona: Oxigen Viena.

Dussel, I. y Southwell, I. (2007). Ante el dolor, ¿̇qué puede la escuela? Recuperado de http://www.me.gov.ar/monitor/nro12/dossier1.htm

Esquerda, M. y Agustí, A. M. (2010). El nen i la mort. Acompañar els infants $i$ adolescents en la pèrdua d'una persona estimada. Lleida: Pagès Editors.

Fernández, I. (2013). El Joan ha mort. Conte i guía per acompañar els nens i els adolescents en el dol la comprensió de la mort. Lleida: Pagès Editors.

González, I. y Herrán, A. (2010). Introducción metodològica a la muerte y los miedos en educación infantil. Tendencias Pedagógicas, 15(1), 124-149

Herrán, A. y Cortina, M. (2006). La muerte y su didáctica. Manual para educación infantil, primaria y secundaria. Madrid: Universitas S.A.

Herrán, A. y Cortina, M. (2008). La educación para la muerte como ámbito formativo: Más allá del duelo. Psicooncología: Investigación y Clínica Biopsicosocial en Oncología, 5(2-3), 409-424.

Herrán, A. y Cortina, M. (2009). La muerte y su enseñanza. Diálogo Filosófico, 75, 499-516.

Herranz, S. (2016). Los niños ante el duelo por la muerte de un ser querido. Recuperado de http://haztua.com/duelo-en-menores/

Kroen, W. (1996). Cómo ayudar a los niños a afrontar la pérdida de un ser querido. Barcelona: Paidós.

Kübler-Ross, E. (2003). Aprender a morir-aprender a vivir. Preguntas y respuestas. Barcelona: Sirpus.

Poch, C. y Herrero, O. (2003). La muerte y el duelo en el contexto educativo. Reflexiones, testimonios y actividades. Barcelona: Paidós.

Ramos Álvarez, R. (2014). Las estrellas fugaces no conceden deseos: programa de prevención, evaluación e intervención por duelo en el contexto escolar. Madrid: TEA Ediciones.

Ransanz, E. (2015). La muerte en el desarrollo de la madurez personal del educando. En A. Cagnolati y J. L. Hernández Huerta (Coords.), Pedagogía ante la muerte. Reflexiones e interpretaciones en perspectivas histórica y filosófica (pp. 161-167). Salamanca: FahrenHouse.

Rodríguez Fernández, I. (2000). Génesis y evolución de las actitudes ante la muerte en la infancia. Cuadernos de Bioética, 1, 113-118.

Serra, X. (2014). I jo, també em moriré? Com es pot ajudar els infants i els joves a conviure amb la pèrdua i la mort de qui estimem. Barcelona: Columna.

Shumba, J., Moyo, G. y Rembe, S. (2015). Bereavement counseling: A dilema for school counselors? International Journal of Science Education, $11(3)$, 277-284. 
Song, H. D. y Kim, K. H. (2015). A study on the development of the prioritzation items for studentintended well-dying education program using DACUM technique. Indian Journal of Science and Technology, 8(25), 1-7.

Turner, M. (2004). Cómo hablar con niños y jóvenes sobre la muerte y el duelo. Barcelona: Paidós.

Zhang, J. y Li, X. (2016). Study of life education for college freshmen in China. Interntional Education Studies, 8(12), 75-80.

\section{Breve CV de los autores}

\section{Anabel Ramos-Pla}

Investigadora en formación de la Universitat Autònoma de Barcelona (España). Además, ejerce profesionalmente como maestra de Educación Primaria y colaboradora en proyectos de investigación del grupo EDO-UdL de la Universitat de Lleida. Pese a su juventud, ya ha realizado diversas publicaciones en referencia a la pedagogía de la muerte y en su vertiente preventiva. Sus principales líneas de investigación están relacionadas con la pedagogía de la muerte y la innovación en las metodologías docentes. ORCID ID: 0000o001-5437-150X. Email: anabelrms@gmail.com

\section{Joaquín Gairín}

Maestro y doctor en Pedagogía y en Psicología. Actualmente, catedrático de Didáctica y Organización en la Universitat Autònoma de Barcelona. Tiene experiencia docente en todas las etapas del sistema educativo, habiendo sido director de centro educativo, responsable de departamento universitario, decano de la Facultad de Educación y responsable del Clúster de la Educación y Formación de la UAB.

Consultor internacional, ha participado en reformas educativas de varios países iberoamericanos en temáticas sobre organización y dirección de centros, evaluación institucional, cambio educativo y escuelas seguras y saludables. Actualmente, es director del grupo EDO (http://edo.uab.es) y coordinador de RedAGE (http://www.redage.org). ORCID ID: 0000-0002-2552-0921. Email: joaquin.gairin@uab.es

\section{Ramón Camats}

Doctor en Filosofia por la Universidad Autónoma de Barcelona. Profesor asociado de la Universidad de Lleida. Departamento de Pedagogía y Psicologia. Autor de varios ensayos de Filosofia del Derecho y de Sociología: "Les emocions del poder" (Premio Josep Vallverdú de ensayo. Lleida). "El llegat d'Antígona" (Premio Josep Vallverdú de ensayo. Lleida). "El clergue i el bufó" (Finalista Premi Assaig Breu revista Idees). ORCID ID: 0000-0003-0042-8886.Email: rcamats@pip.udl.cat 